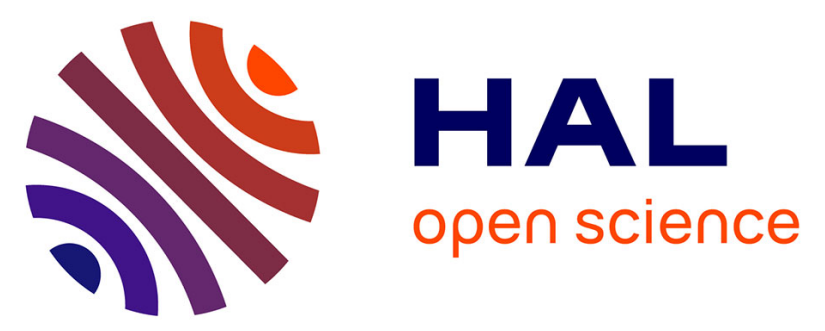

\title{
ALMA 2015: Sea Trial Of An Underwater Target Localization Technique Using Hausdorff Distance
}

Pedro Martins de Magalhães, Xavier Cristol, Cornel Ioana, Dominique Fattaccioli, Jerome I. Mars

\section{- To cite this version:}

Pedro Martins de Magalhães, Xavier Cristol, Cornel Ioana, Dominique Fattaccioli, Jerome I. Mars. ALMA 2015: Sea Trial Of An Underwater Target Localization Technique Using Hausdorff Distance. OCEANS 2018 - OCEANS '18 MTS/IEEE. Ocean Planet - It's our home., May 2018, Kobe, Japan. hal-01802569

\section{HAL Id: hal-01802569 \\ https://hal.science/hal-01802569}

Submitted on 29 May 2018

HAL is a multi-disciplinary open access archive for the deposit and dissemination of scientific research documents, whether they are published or not. The documents may come from teaching and research institutions in France or abroad, or from public or private research centers.
L'archive ouverte pluridisciplinaire HAL, est destinée au dépôt et à la diffusion de documents scientifiques de niveau recherche, publiés ou non, émanant des établissements d'enseignement et de recherche français ou étrangers, des laboratoires publics ou privés. 


\title{
ALMA 2015: SEA TRIAL OF AN UNDERWATER TARGET LOCALIZATION TECHNIQUE USING HAUSDORFF DISTANCE
}

\author{
Pedro E. Magalhães ${ }^{1,2}$, Xavier Cristol ${ }^{1}$, Cornel IOANA ${ }^{2}$, Dominique FATTACCIOLI ${ }^{3}$ and Jérôme Mars ${ }^{2}$ \\ Member IEEE,
}

2 Thales Underwater Systems, Sophia Antipolis, France

1 Univ. Grenoble Alpes, CNRS, G-INP, GIPSA-Lab, 38000-France

3 DGA Naval Systems, Toulon, France

\begin{abstract}
$\square \square\|\| \square-$ The fundamental and practical problem of passive localization in range and depth, of an acoustic underwater source is addressed, with application to an at-sea experiment. We propose and try a new matching method based on a metric called as Hausdorff distance as a cost-function to be minimized, in order to perform the localization inversion. The data set analyzed here was collected during the DGA campaign ALMA 2015, which took place in a shallow water environment of the southern coast of France. Acoustic data were measured over a $10 \mathrm{~m}$-high vertical linear array (VLA), composed of 64 hydrophones. The 2-D localization, in range and depth, is performed by matching the patterns of time difference of arrival (TDOA), between respectively observed and modeled sequences. Several variants of the Hausdorff Distance are applied, firstly separately in each single hydrophone, and then combined in order to improve the localization accuracy, reducing the ambiguity either is depth and in range. The performance is evaluated in terms of the localization accuracy of the proposed method, in a context of passive localization with a cooperative system considering a motionless target. Very satisfactory performance and accuracy are obtained.
\end{abstract}

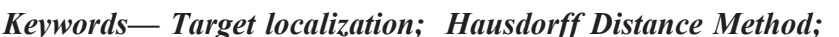

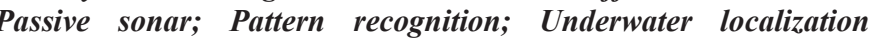

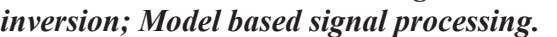

\section{INTRODUCTION}

Over the last decade, an enduring interest has being growing in passive sonar localization and tracking of underwater acoustic targets, using intercepted transient pulses. However this still challenging problem remains a constant research theme, being of a high strategic value for navy operations, most specific in protection of coastal areas.

The context of shallow water combined with long target range produces signals reaching the sonar along multipath with small grazing angles of arrival, very close one to each other in angle and in travel time; a tremendous obstacle for achieving an accurate ranging of the target is the complex behavior of the sound channel, which features many interactions with complex boundaries (rough seabed, rough moving sea surface). Unstationary more-or-less random interference patterns, which can be either constructive (gain) or destructive (fading), impact the performance of signal processing. The signal received by each sonar sensor is assumed to be the sum of copies of the transmitted waveform from the target. It contains information about the specific range and depth of the target through the characteristics of the different propagation paths, and first of all through their different arrival times and arrival angles. Hence the target location information can be inferred from two widely used parameters: Time Difference Of Arrival (TDOA) and/or Angles Of Arrival (AOA). The most common technique for underwater localization consists of firstly predicting the received acoustic signals and the corresponding TDOA and/AOA modeled, with the help of an acoustic propagation models derived from the wave equation (rays theory, Normal modes, parabolic equation, multipath expansion, wavenumber integration.) for a set of possible target locations; these simulations sharply depend on the accuracy of the a priori knowledge of the undersea environment. The second step consists of finding the target location that produces the best matching between the modeled signal parameters and the measured ones; this best matching may be searched and achieved using different optimizations methods and criteria at the literature. The combination of several hydrophones improves and makes more robust the localization processing and its accuracy by making use of spatial diversity, as a remedy to the problem of multipath interference and fading.

Significant data-to-model mismatch can result even in a relatively well characterized environment, a wrong location of the target.

Uncertainties in environmental parameters may affect all features of the marine medium: sound speed profile (fronts, upwelling, tides, internal waves, currents and turbulence) influences the times and angles; seabed topography (microtopography, ripples) affects bottom reflection angles; sea bottom geo-acoustic properties like sediment type or layering of the seafloor affect reflection coefficient and may impact the detectability of late arrivals; the sea surface affected by gravity waves is an intrinsically random boundary and affects both reflection coefficients and arrival angles. In many cases, even a small variation in one of these items can change dramatically the propagation paths. Target localization in an operational context cannot rely on too complex time-consuming propagation models, requiring practically unavoidable environmental data; for remaining close to such practical situations, the representation of the shallow water medium involved in our localization scheme, was deliberately highly simplified as a range independent acoustic propagation model, calling for a mean constant Sound Speed Profile (SSP) and for a nominal flat seafloor with a local averaged depth. 
Our localization technique relies on recognizing the sequence of TDOA over all sensors of an array. When the signal radiated from the source is broadband and when the waveform parameters are known or can be estimated, the most popular method for estimating the TDOA is a so-called matched filtering (correlation of signal with estimated waveform). The TDOA can also be obtained by other methods such as described in: [1], [2] and [3]. The variance of the estimated TDOA depends on SNR, pulse bandwidth and frequency [4]. The localization can then be performed by using matched mode processing [5] or matched field processing [6] such as conventional beamforming (Bartlett) and high-resolution algorithms (adaptive beamforming), which include: maximum likelihood [7], MUSIC and derivatives [8], ESPRIT [9], and feature extraction methods [10]. High resolution algorithms mainly commonly use hydrophone arrays to localize a target.

Localization may be understood as an optimization problem, where the model-to-observation discrepancy, as measured by some cost-function, must be minimized. The proposed method makes use of Hausdorff Distance (HD) as such a cost-function. The HD technique outlined in this paper has been widely used in image processing and pattern recognition, in many different fields like radar and satellite image processing[11], ship positioning by combining radar images with satellite images [12], speech recognition [13], video recognition, computer vision [14], underwater vehicle classification [15] [16], and sidescan sonar [17]. In the context of underwater localization, it was first used: in active sonar by Mours et al [18], and in passive sonar by Magalhaes et al [19] in a tank experiment, demonstrated that the HD could also be applied in a single hydrophone configuration.

The main advantage of the HD as a cost function to be minimized is that it takes into consideration the spatial proximity of each individual point in the observation and in a rasterized grid of simulated points. It is far more robust to the environmental mismatch when compared with other wellknown localization techniques, such as the Matched Field Processing (MFP), where even a small mismatch between the two sets of observed and modeled points may preclude from matching; this would strongly affect the accuracy of the location. The basic difference between these two methods is that the MFP evaluates the position of the target based on the maximum value of the output correlator of the observed impulse response, which contains the information about the Time Differences Of Arrival (TDOA) and Angles of Arrival (AOA) of all ray paths. The HD distance quantifies the similarity between the two measured and modeled sets, TDOA and/or AOA). The correct target location is then identified as the output from a global search for the minimal value, corresponding to a maximal match between those sets, theoretically being zero if both sets are identical. In presence of an environmental mismatch, an error would be introduced in the modeled sets, and induce a corresponding error in the estimated location of the target; however it seems that the impact of the modeling error on results from the HD cost function is far less critical than for the MFP correlation. A second advantage of $\mathrm{HD}$ is the short computational runtime, again comparing with the MF. The HD also offers an opportunity for a hierarchical cell decomposition strategy, defining a preliminary large cell grid for a rough first global search, and then refining the mesh size for a higher final accuracy.

This article consists of five sections. Section II describes the environment and the instruments of the ALMA 2015 sea trial setup. Section III describes the three proposed cost-functions based on variants of the HD and its applicability to underwater localization. Section IV presents the results of our technique applied to ALMA 2015 experiment. Section V gives conclusions and evokes future work.

\section{THE ALMA 2015 EXPERIMENT}

Experimental acoustic and environmental data were collected during the three days of ALMA 2015 campaign, making use of the modular array-and-source system ALMA (Acoustic Laboratory for Marine Applications) [20], designed and managed by DGA Naval Systems. It took place in October 2015 , in a shallow water environment off the southern coast of France, near the harbor of Toulon. Fig. 1 presents the location of the acoustic projector and of the receiving 10m-high array (64 vertical linear passive array hydrophones (VLA), distributed in depth from $52.5 \mathrm{~m}$ to $62.3 \mathrm{~m}$. The seafloor was roughly flat, with a depth about $100 \mathrm{~m}$, with a sediment cover constantly sandy or gravelly-sandy. During the processed sequence, the calm sea roughness height was about $0.1 \mathrm{~m}$ (sea state 1 ). The source-toreceiver range was about $9.0 \mathrm{~km}$. The source, moored at a depth of $56.2 \mathrm{~m}$, transmitted sequences featuring different narrow- and wide-band codes, among which we considered only Linear Frequency Modulations (LFM) over the of 4 to $6 \mathrm{kHz}$ frequency band; the acoustic signals were sampled at $48 \mathrm{kHz}$. We have analyzed 50 signals over about two hours, with one transmission every three minutes.

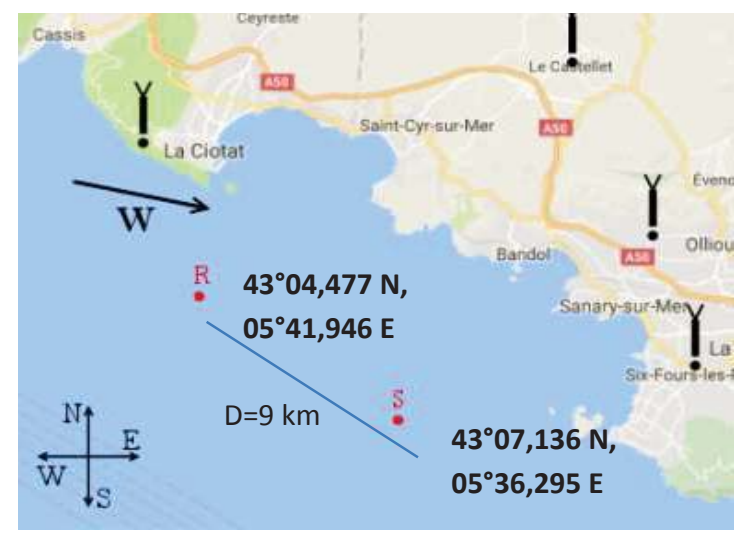

Fig. 1.Top view of the experimental setup measurements region.

\section{LOCALIZATION METHOD}

\section{1) MaXimum HaUSDORfF Distance}

The Maximum Hausdorff Distance (MaxHD), introduced by Huttenlocher [21] in the field of image processing, is a technique to measure the degree of similarity among different sets of points over a metric space. Here we invoke the HD for evaluating the distance between two "1D images", namely two sequences of respectively modeled and measured TDOA, giving an interesting measure of their mutual suitable proximity.

Two bounded non-empty subsets $\mathrm{A}$ and $\mathrm{B}$ of an metric space are considered, one being the measured sequence of TDOA 
$\mathrm{R}_{\mathrm{T}}=\left\{\mathrm{r}_{1}, \mathrm{r}_{2}, \mathrm{r}_{3}, \ldots, \mathrm{r}_{\mathrm{x}}\right\}$, and the other one the modeled sequence of TDOA $S_{\mathrm{T}}=\left\{\mathrm{s}_{1}, \mathrm{~s}_{2}, \mathrm{~s}_{3}, \ldots, \mathrm{s}_{\mathrm{y}}\right\}$. Each point in one of the two sequences is assigned to its nearest neighbor in the other sequence, and the distance between those selected points is the Euclidean norm $\|$.$\| . This way, a sequence of point-to-point$ distances is obtained; the roles of the modeled and measured TDOA may be exchanged, resulting in two different sequences of distances.

The final step for defining the HD consists of selecting the largest one among these minimal distances, which is the largest departure between the two sets of points. Three variants exist, depending on the sequence used for extracting the maximum: backward and forward sequences, backward alone, and forward alone. Typically, the equation most commonly presented in the literature is the maximum bidirectional HD, defined as:

$$
\mathrm{H}\left(\mathrm{R}_{\mathrm{T}}, \mathrm{S}_{\mathrm{T}}\right)=\max \left\{\mathrm{h}\left(\mathrm{R}_{\mathrm{T}}, \mathrm{S}_{\mathrm{T}}\right), \mathrm{h}\left(\mathrm{S}_{\mathrm{T}}, \mathrm{R}_{\mathrm{T}}\right)\right\}
$$

In this paper we will use the notations $\mathrm{h}\left(\mathrm{R}_{\mathrm{T}}, S_{\mathrm{T}}\right)$ and $\mathrm{h}\left(\mathrm{S}_{\mathrm{T}}\right.$, $\mathrm{R}_{\mathrm{T}}$ ) for the forward and backward HD respectively. Those two "pseudo-distances" are not symmetrical in general, depending on the number of points at each subset, and the combinations with the near neighbors themselves:

$$
\begin{aligned}
& \mathrm{h}\left(\mathrm{R}_{\mathrm{T}}, \mathrm{S}_{\mathrm{T}}\right)=\max _{\mathrm{r} \in \mathrm{R}}\left[\min \left(\left\|r_{i}-s\right\|\right)\right] \quad \mathrm{i}=\{1,2,3, \ldots, \mathrm{x}\} \\
& \mathrm{h}\left(\mathrm{S}_{\mathrm{T}}, \mathrm{R}_{\mathrm{T}}\right)=\max _{\mathrm{s} \in \mathrm{S}}\left[\min \left(\left\|s_{i}-r\right\|\right)\right] \quad \mathrm{j}=\{1,2,3, \ldots, \mathrm{y}\}
\end{aligned}
$$

The MaxHD reaches its minimal value when every point of the modeled TDOA sequence is close to some point of the measured TDOA sequence. If the modeled environment is close enough to the experimental setup environment, the simulations of acoustic propagation models should give, for the correct target location, the same sequence of TDOA, with an ideal HD equal to 0 , or practically reaching its minimum. The best matching (localization) is then performed through a global search for the minimal output of MaxHD between the measured TDOA sequence and the modeled TDOA sequence where a sampling of source location in depth and range is tried.

The MaxHD tolerates small variations in time at the set of TDOAs, and it is unaffected by variations in absolute phase or initial delay, being in that sense an incoherent process. The disadvantage of only selecting the farthest distance in the definition of MaxHD appears in presence of outliers; those extra-points un-legitimately dominate the final MaxHD, and may be responsible for huge mismatches. Two variants of the basic MaxHD, presented in the following, mitigate this specific problem.

In the following, this paper will consider only the backward Hausdorff Distance; the reason for this choice is that the number of points in the set of modeled TDOA in this experiment was less than the number of the measured TDOA. this is important because of avoiding the missing point problem, i.e. points that were not modeled after over the $15 \mathrm{~ms}$ interval after the first path arrival; This number of ten came from a previous analysis in a realistic operational scenario, related with the average transmitted source power signal and the level of detection of the received array, provided a SNR allowing the detection of a source at a specified range.

\section{2) Mean Hausdorff Distance}

A first variant of the MaxHD, proposed by Dubuisson, is the Mean Hausdorff Distance [22], where the average of the Euclidian distances is taken instead of its maximum. The MeanHD is defined as:

$$
\overline{\mathrm{h}}\left(\mathrm{S}_{\mathrm{T}}, \mathrm{R}_{\mathrm{T}}\right)=\frac{1}{\mathrm{Y}} \sum_{\mathrm{j}=1}^{\mathrm{Y}}\left[\min _{s \in S}\left(\left\|s_{i}-r\right\|\right)\right] \quad \mathrm{j}=\{1,2,3, \ldots, \mathrm{y}\}
$$

The main advantage of the MeanHD is that all points contribute to the final measure of similarity, which ensures that the other closest points will be take into account, and not only the ones that are the farthest one to each other and which can be outliers. In other words, the big mismatch which could arise from those outliers, is divided by the number of the subset points and in this way the error can be reduced.

\section{3) MEDiAn HAUSDORFF DistANCE}

The second variant, called the Median Hausdorff Distance (MedHD), was also proposed by Dubuisson [22], Instead of the maximum or the average of the Euclidean distances, their median is adopted:

$$
\mathrm{h}^{50 \text { th }}\left(\mathrm{S}_{\mathrm{T}}, \mathrm{R}_{\mathrm{T}}\right)=\mathrm{K}^{50 \text { th }}\left\lfloor\min _{s \in S}\left(\left\|s_{i}-r\right\|\right)\right\rfloor \mathrm{j}=\{1,2,3, \ldots, \mathrm{y}\}
$$

The main advantage of this variant compared with the others two previous, is its stronger robustness to the outlier artifacts, due to the elimination of the largest distances; however, if no extra-point, the MedHD does not take benefit of the total amount of available points, because $50 \%$ of points in principle do not contribute to the final result.

\section{RESUlTS OF THE HD TECHNIQUES}

The signal transmitted from the source reaches each sensor along a cluster of multipath arrivals with different energies, and very close arrival time delays and angles, resulting in overlaps and interference. When considering a single sensor, these small differences in time or angle can generate an error or ambiguity either in target range or in depths. The spatial diversity among the sensors of the quite high array $(10 \mathrm{~m})$ and the coherent structure of the signals may help in relaxing these unfortunate consequences of this dense clustering. The combination of all hydrophones should reduce the ambiguity generated by the interferences and overlaps, and improve the target localization. In our first trial, we use only TDOA; the combination of all sensors is also important because it stabilizes the estimates of the measured TDOA sequence to be matched with the modeled TDOA sequences. Table 1 shows the result of our three various HD localization techniques during the analyzed set of 50 pings.

Table I .Percentage of pings with localization errors less than $1 \mathrm{~km}$ error in range and less than $10 \mathrm{~m}$ in depth (True solution: range $9 \mathrm{~km}$; depth $56 \mathrm{~m}$ ) 


\begin{tabular}{ccc}
\hline \hline \% of the pings localized & range $(\%)$ & depth $(\%)$ \\
\hline Maximum Hausdorff Distance & 42 & 36 \\
\hline Mean Hausdorff Distance & 68 & 50 \\
\hline Median Hausdorff Distance & 44 & 60 \\
\hline \hline combination of Hausdorff Distances & 76 & 64 \\
\hline \hline
\end{tabular}

The best result among the tested variants is obtained with the MeanHD, which was able to correctly localize the position of the targetin range in $68 \%$ of considered transmissions, and localize in depths in $50 \%$ of transmissions, considering an error less than $1 \mathrm{~km}$ in range and less than $10 \mathrm{~m}$ in target depth. These errors are departs of about $10 \%$ around the correct target location. According to operational navy specialist, one considers a localization as sufficiently correct when the error is less than $20 \%$ of the target position;we reach the half of this critical value. The next step was to evaluate a fourth cost function: the combination of all three HD variants. The improvement brought by this combination is significant: the target is correctly localized in $76 \%$ of pings for error less than $1 \mathrm{~km}$ in range and in $64 \%$ for error less than $10 \mathrm{~m}$ in depth. The idea of using a combination of two variants of the HD came from the awareness of a possible mismatch between the 2 sets of points, which can generate ambiguities and finally wrong target position. Since each different variant performs different combinations, we may expect that secondary lobes occurs in different positions. As a result, the combinations can be used as a remedy to mitigate this particular problem.

The last improvement came from using mean spatial filtering with the window four-by-four, after the combination of the 64 hydrophones. Each hydrophone has its own measured sequence of TDOA, which may feature a small time mismatch resulting in a small spatial mismatch of target location. The combination of the 64 hydrophones takes advantages of the spatial diversity of the vertical linear array; this spatial filtering allows us to evaluate the information at the target's neighborhood.

Each variant of the Hausdorff method features has its own histogram of localization results based on measures of error generated by the different matching of TDOAs. The Fig.2 displays the histograms of estimated target range and depth using the combination of the three HD variants techniques: a sharp dominant peak clearly appears at the correct depth and range locations, with far lower secondary ambiguity maxima.
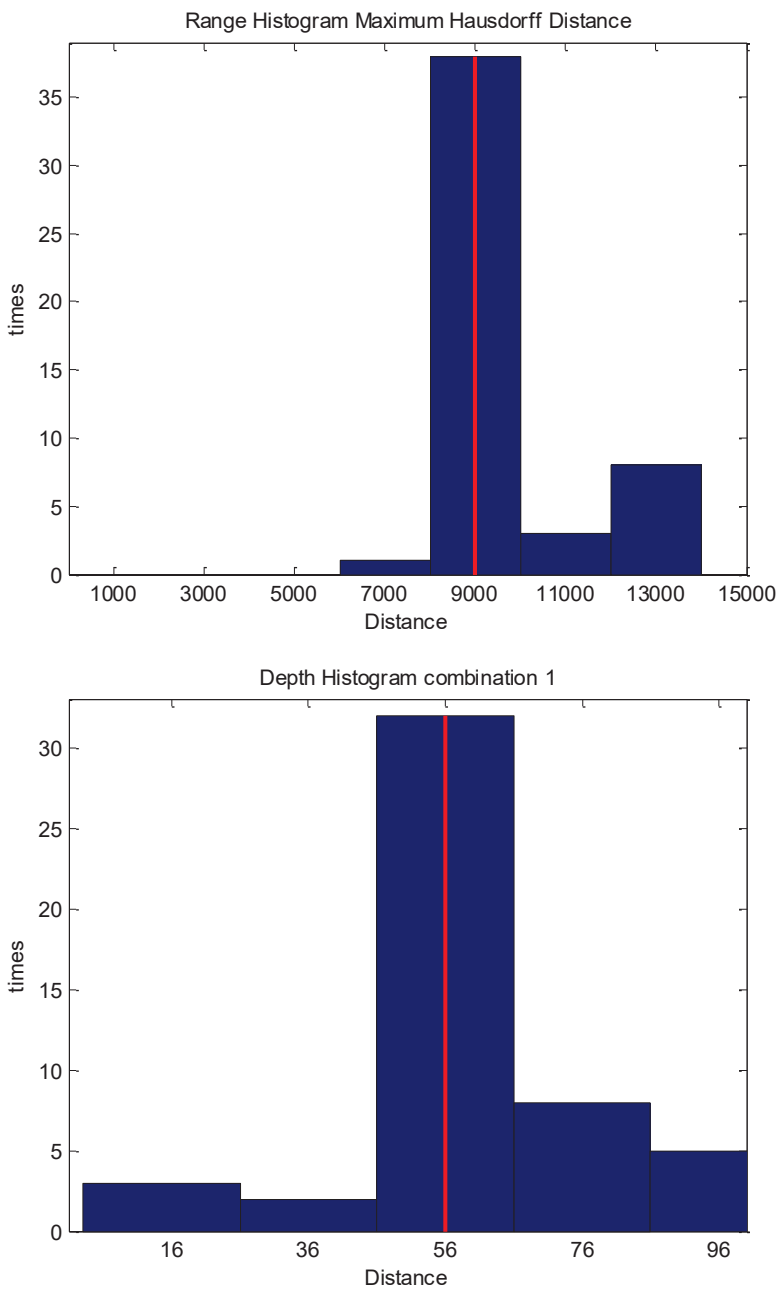

Fig. 2.HD combination error histogram in range (top), and in depth (bottom)

\section{CONClusion}

We have developed and tried several variants of an acoustic target localization technique relying of the Hausdorff Distance as a cost-function to be minimized when comparing observations with model outputs for a sampling of target range and depth. These methods were validated on real data involving a part of the signals collected in October 2015, in a shallow Mediterranean environment, during the DGA experimental campaign ALMA 2015. Target localization using these experimental data was challenging to underwater localization due to its difficult configuration: shallow water and long range, plus both transmitter and receivers located in mid-depth column waters. This generates a strong interfering cluster of multiple surface and bottom-reflected arrivals, close one to each other in time and angle, which are often difficult to separate within the signal temporal resolution capacity. The second difficulty was the choice of a highly simplified modeled channel for the inverse problem, due to the fact that we wanted to test our technique in a worst case scenario, close to operational situations, and to test the robustness of the proposed method in combating the environmental mismatch: the irregularities and movements of 
the sea surface and bottom are disregarded; the SSP fluctuations are also ignored. The Hausdorff Distance techniques, historically used in the fields of image processing and shape recognition, seems to be a robust incoherent model based signal processing in order to perform the localization inversion, as demonstrated in a real at sea experiment, in a context of cooperative passive underwater localization. A significant improvement on the localization accuracy can be reached by using the spatial filtering to mitigate the information on the target's neighborhood. Overall, considering the theoretical and experimental feasibility, the proposed method has shown very satisfactory for localizing a target, with a good operational precision.

\section{ACKNOWLEDGMENT}

This work was supported by Thales Underwater Systems and DGA (Direction Générale de l'Armement). The authors wish to thank Mr. Dominique FATTACCIOLI and Mr. Gaultier REAL (DGA Naval Systems) for their contributions in collecting the data during the 3 days experiment and in providing details of the experiment itself, Mr. Bruno CHALINDAR (Thales Underwater Systems) in providing the modeled TDOA based on the Thales ray propagation model RAMSES, and $\mathrm{Mr}$. Iannis BENNACEUR (Thales Underwater Systems) for helpful information resulting from a previous analysis of the ALMA 2015 experiment and for the generation of pictures presented in this paper. The authors also thank the captain and the crew of the COMEX research vessel Janus, which sailed during the ALMA 2015 campaign.

\section{REFERENCES}

[1] J. Hassab and R. Boucher, "Optimum estimation of time delay by a generalized correlator," IEEE Transactions on Acoustics, Speech, and Signal Processing, pp. 373-380, 1979.

[2] C. Knapp and G. Carter, "The generalized correlation method for estimation of time delay," IEEE Transactions on Acoustics, Speech, and Signal Processing, pp. 320-327, 1976.

[3] G. Carter, "Time delay estimation for passive sonar signal processing," IEEE Transactions on Acoustics, Speech, and Signal Processing, pp. 463-470, 1981.

[4] A. Quazi, "An overview on the time delay estimate in active and passive systems for target localization," IEEE

Transactions on Acoustics, Speech, and Signal Processing, pp. $527-533,1981$.

[5] A. B. Baggeroer, W. A. Kuperman and P. N. Mikhalevsky, "An overview of matched field methods in ocean acoustics," IEEE Journal of Oceanic Engineering, pp. 401-424, 1993.

[6] H. Schmidt, A. B. Baggeroer, W. A. Kuperman and E. K. Scheer, "Environmentally tolerant beamforming for high- resolution matched field processing: Deterministic mismatch," The Journal of the Acoustical Society of America, 1990.

[7] N. E. Hurt, "Maximum likelihood estimation and MUSIC in array localization signal processing: A review,"

Multidimensional Systems and Signal Processing, p. 279-325, 1989.

[8] J. M. Ozard, "Matched field processing in shallow water for range, depth, and bearing determination: Results of experiment and simulation," The Journal of the Acoustical Society of America, 1989.

[9] Q. Zhang and J. Huang, "Joint estimation of DOA and timedelay in underwater localization," in IEEE International Conference on Acoustics, Speech, and Signal Processing. Proceedings, Phoenix, 1999.

[10] K. Kim, W. Seong and C. Lee, "Matched field processing: analysis of feature extraction method with ocean experimental data," in Proceedings of the 2004 International Symposium on Underwater Technology, 2004.

[11] P. Bustos, F. Donoso, A. Guesalaga and M. Torres, "Matching Radar and Satellite Images Employing the Hausdorff Distance for Ship Positioning and Trajectory Estimation," in 2006 International Conference on Image Processing, Atlanta, 2006.

[12] M. Torres-Torrit and A. Guesalaga, "Automatic ship positioning and radar biases correction using the hausdorff distance," in 10th International Conference on Information Fusion, Quebec, 2007 .

[13] A. Andreev and N. Kirov, "Word Image Matching Based on Hausdorff Distances," in 10th International Conference on Document Analysis and Recognition, 2009.

[14] S. H. Kim and R.-H. Park, "An efficient algorithm for video sequence matching using the modified Hausdorff distance and the directed divergence," IEEE Transactions on Circuits and Systems for Video Technology, pp. 592-596, 2002.

[15] H. Peyvandi, B. Fazaeefar and H. Amindavar, "Determining class of underwater vehicles in passive sonar using hidden Markov model with Hausdorff similarity measure," in Proceedings of 1998 International Symposium on Underwater Technology, Tokyo, 1998.

[16] T. L. Hemminger and Y.-H. Pao, "Detection and classification of underwater acoustic transients using neural networks," IEEE Transactions on Neural Networks, pp. 712-718, 1994.

[17] S. Reed, Y. Petillot and J. Bell, "Automated approach to classification of mine-like objects in sidescan sonar using 
highlight and shadow information," IEE Proceedings - Radar, Sonar and Navigation, pp. 48-56, 2004.

[18] A. Mours, C. Ioana, J. Mars, N. Josso and Y. Doisy, "Targetdepth estimation in active sonar: Cramer-Rao bounds for a bilinear sound-speed profile," The Journal of the Acoustical Society of America , 2016.

[19] C. I. J. M. a. X. C. P. E. M. de Magalhães, "Hausdorff distance applied on real data experiment for underwater localization," in OCEANS 2017, Aberdeen, 2017.

[20] G. R. Dominique Fattaccioli, "The DGA "ALMA" Project: an overview of the recent improvements of the system capabilities and of the at-sea campaign ALMA-2016," in UACE 2017, Greece, 2017.

[21] D. P. Huttenlocher, G. A. Klanderman and W. J. Rucklidge, "Comparing images using the Hausdorff distance," IEEE Transactions on Pattern Analysis and Machine Intelligence, pp. 850-863, 1993.

[22] M. P. Dubuisson and A. K. Jain, "A modified Hausdorff distance for object matching," in Proceedings of 12th International Conference on Pattern Recognition, Jerusalem, 1994.

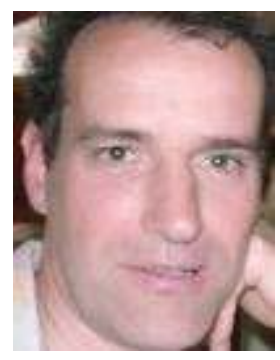

Jérôme Mars received his Masters (1986) in Mechanics and Geophysics from University Joseph Fourier (currently University Grenoble Alpes), and his $\mathrm{PhD}$ in Signal Processing (1988) from the Institut National Polytechnique de Grenoble. He was with the Centre des Phénomènes Aléatoires et Géophysiques de Grenoble (CEPHAG) from 1989 to 1992, with the Materials Sciences and Mineral Engineering (MSME) Department of University of California, Berkeley, from 1992 to 1995, and with Laboratory of Images and Signals (LIS) from 1995 to 2007 . He is a Professor (C-Ex) with Grenoble Institute of Technology (INP), Grenoble, France. He is currently Head of the Grenoble Images Speech Signals and Automatics Laboratory (GIPSA-Lab, UMR 5216, UGA, CNRS, G-INP). His research interests cover statistical signal processing and source separation. In particular, his latest research is mostly focused on the field of wave propagation (Underwater Acoustics, Geosciences, Tomography). With more than 80 peer-reviewed publications and 200 international conference attendances, he is involved in the supervision of more than $30 \mathrm{PhD}$ students who are related to his research interests. He is a member of IEEE.

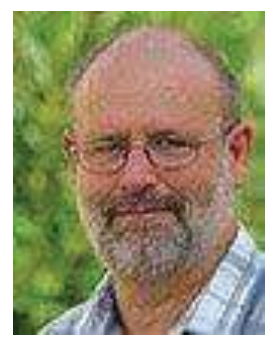

Xavier Cristol received engineering degrees at the Ecole Centrale de Lyon in 1984 and his PhD in Acoustics in 1987. Since 1988, he works at Thales Underwater Systems as expert in underwater acoustics. His current interests include acoustic propagation, reverberation and noise in oceanic environments.

Cornel Ioana received the Dipl.-Eng. degree in electrical engineering from the Romanian Military

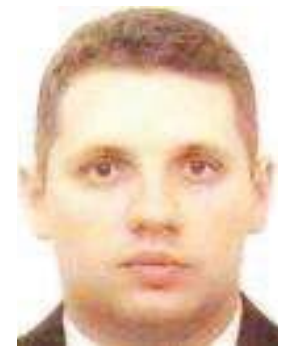

Technical Academy of Bucharest in 1999 and the M.Sc. degree in telecommunication science and the $\mathrm{Ph} . \mathrm{D}$. degree in the electrical engineering field, both from University of Brest-France, in 2001 and 2003 , respectively. Between 1999 and 2001, he activated as a Military Researcher in a research institute of the Romanian Ministry of Defense (METRA), Bucharest). Between 2003 and 2006, he worked as Researcher and Development Engineer in ENSIETA, Brest-France. Since 2006, he is an Associate Professor-Researcher with the Grenoble Institute of Technology/GIPSA-lab. He is co-head of the SIGMAPHY (Signal Image Physics) research team of GIPSA-lab. His current research activity deals with the signal processing methods for energy and environment applications. His scientific interests are non-stationary signal processing, natural process characterization, radar and acoustic systems and distributed sensors monitoring in energy and environment systems.

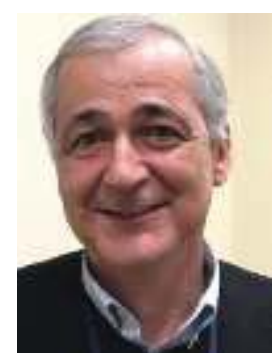

Dominique Fattaccioli received is $\mathrm{PhD}$ in Theoretical Physics (1987) from Paris University (Paris 6 Pierre \& Marie Curie). He is currently senior scientific expert in underwater acoustics at DGA (Direction Générale de l'Armement), and Deputy Head of the Underwater Warfare Department at DGA Naval Systems in Toulon, France.

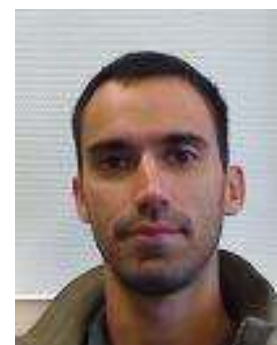

Pedro E. Magalhães was born in Rio de Janeiro Brazil, in 1987. He received his BSc and MSc degrees in Electric and Telecommunication Engineering from the Fluminense Federal University (UFF), Niteroi, Brazil, in 2012 and 2015, respectively. He is currently doing his PhD in Acoustical Engineering at GrenobleAlpes University, Grenoble, France.

From 2012 to 2015 , he was a Researcher on a project with the Brazilian Navy on underwater communication systems and signal processing.

His present research interests include underwater passive source localization and underwater acoustics propagation. 